\title{
Le commerce des idées
}

La création conceptuelle s'alimente le plus souvent de circulations invisibles entre les disciplines, par emprunts aux unes et aux autres, par détournements et réappropriations, sorte de bricolage aventurier qui suppose beaucoup de travail et de rigueur mais aussi beaucoup de prudence dans la quête d'universalité. En quelque sorte, un commerce des idées que le "Groupe des Dix », qui fait l'objet d'un dossier dans ce numéro, illustre à merveille. Pendant 8 ans, à partir de 1968, des intellectuels français, issus d'horizons disciplinaires divers, ont échangé, au gré d'agapes mensuelles, sur des questions qui sont au cœur de notre projet éditorial : une interdisciplinarité à l'épreuve de la complexité, de l'évolution des relations sciences/société, des perspectives de développement à long terme, de la prise en compte de l'environnement...

La dispersion de ce groupe informel coïncide - et contraste - avec une institutionnalisation croissante de la recherche qui se concrétise, dans les années 1970, par la montée en puissance de la DGRST $^{1}$ qui, au travers d' " actions concertées » indépendantes de la programmation des établissements de recherche et préfigurant en cela le futur ministère de la recherche, questionne, elle aussi, les notions de dynamiques, de complexité et de modélisation. C'est dans cette trajectoire de pensée, à l'ombre des institutions ou pas, que se sont inscrites et construites l'association NSS-Dialogues et la revue Natures Sciences Sociétés, au début des années 1990. Certes, il s'agit là d'un tout autre contexte, mais pas au point de rompre une continuité intellectuelle qui ne se dément pas, comme nous l'avons rappelé à l'occasion des 25 ans de la revue et de l'association qui la porte ${ }^{2}$. Curieusement, si les questions et le contexte ne sont plus les mêmes, les réflexions développées dans la revue, comme en écho à des préoccupations du Groupe des Dix, restent pertinentes : formatage de la pensée et de la conduite des recherches par les cadres disciplinaires et les intérêts économiques, difficultés à mener une recherche en société en prise avec les décideurs et les citoyens entre vulgarisation et instrumentation, nécessité d'un regard critique sur la

\footnotetext{
${ }^{1}$ Délégation générale de la recherche scientifique et technique, sous l'autorité du Premier ministre.

${ }^{2}$ Billaud J.-P., Hubert B., Vivien F.-D., 2018. Natures Sciences Sociétés : 25 ans et toujours d'actualité, Natures Sciences Sociétés, 26, 1, 1-2, https://doi.org/10.1051/nss/2018027.
}

construction scientifique des problèmes de société, équilibre difficile entre excellence et pertinence...

«Une démarche de recherche construite en assemblant de façon méthodique des connaissances, des points de vue, des techniques de travail provenant de disciplines scientifiques différentes », telle est la définition de l'interdisciplinarité proposée par Marcel Jollivet et Jean-Marie Legay ${ }^{3}$. Que ce soit sur le plan théorique ou de la démarche, l'interdisciplinarité a été l'objet de nombreux travaux, dont certains ont été au cœur de la production éditoriale de la revue. Entre 2004 et 2008, constituant un dossier "Interdisciplinarité », NSS a publié une vingtaine de textes faisant état de la manière dont les grands organismes publics de recherche (Cirad, CNRS, Inra, IRD...) et leurs programmes ou départements (le Programme environnement, vie et sociétés [PEVS] du CNRS, le département Systèmes agraires et développement [Sad] de l'Inra), mais aussi les disciplines (sciences de la vie, droit, agronomie...), les sociétés savantes (Académie d'agriculture...) s'emparaient de ces enjeux. Le contexte institutionnel a cependant considérablement évolué au cours de ces dix dernières années au plan national, avec la création de l'Agence nationale pour la recherche, de l'Aeres ${ }^{4}$, des pôles de recherche et d'enseignement supérieur (Pres) puis des Communautés d'universités et établissements (Comue), le lancement des différents Programmes d'investissements d'avenir (PIA) conduisant au regroupement d'établissements d'enseignement supérieur et de recherche sous la forme d'Idex et d'I-Site, sans même parler des Labex, Equipex et autres Instituts Convergence ${ }^{5}$. La course aux financements publics et privés, l'organisation de la recherche en mode projet, la montée en puissance du ranking ont profondément modifié la manière de faire de la recherche et de produire des

\footnotetext{
${ }^{3}$ Jollivet M., Legay J.-M., 2005. Canevas pour une réflexion sur une interdisciplinarité entre sciences de la nature et sciences sociales, Natures Sciences Sociétés, 13, 2, 184-188, https://doi.org/10.1051/nss:2005030.

${ }^{4}$ Devenue en 2013 le Haut Conseil de l'évaluation de la recherche et de l'enseignement supérieur (Hcéres).

${ }^{5}$ Arnauld de Sartre X., Petit O., 2018. Une nouvelle géographie de l'enseignement supérieur et de la recherche: rapprochements, concentrations, tensions... et opportunités?, Natures Sciences Sociétés, 26, 3, 255-256, https://doi.org/ $10.1051 / \mathrm{nss} / 2018049$.
} 
connaissances, du moins dans certains domaines qui n'avaient pas été encore en prise avec ces enjeux. L'interdisciplinarité entre natures et sociétés se trouve ainsi confrontée à de nouveaux défis, face aux réorganisations institutionnelles du milieu de la recherche en France.

Aussi avons-nous ouvert un nouveau champ de réflexion afin de mieux saisir les effets de ces dernières évolutions, pas tant sur l'interdisciplinarité en soi, qui fait de plus en plus souvent l'objet d'injonctions ancillaires dans ces transformations institutionnelles, que sur les tenants cognitifs et organisationnels du projet interdisciplinaire. Ce chantier s'est ainsi organisé en quatre groupes de questionnement au sein du comité de rédaction de NSS, ouverts aux membres de l'association et à d'autres collègues intéressés. Nous en présentons ici les principaux éléments :

- Comment les différents acteurs qui ont vécu ces transformations récentes se positionnent-ils et quel regard portent-ils sur la manière dont leurs institutions (disciplines, organismes de recherche, sociétés savantes...) ont mobilisé l'interdisciplinarité dans leurs discours, mais aussi dans leurs actes? Comment gèrent-ils les injonctions contradictoires faisant de la recherche académique disciplinaire pointue le sceau de l'excellence, alors même que l'interdisciplinarité est plus que jamais revendiquée et attendue dans la soumission de projets, par exemple? C'est à quoi s'intéresse un premier groupe qui interrogera les responsables des organisations et de la politique de la recherche (conditions de programmation, taille et composition des collectifs, conséquences sur les recrutements et l'évaluation, etc.).

- Dans le même temps, dans un contexte d'internationalisation de la recherche, des collectifs interdisciplinaires organisés sous forme de communautés épistémiques (Resilience Alliance, Political Ecology, Ecological Economics...) redéfinissent, voire même s'abstraient des frontières disciplinaires. Ils revendiquent l'interdisciplinarité pour appréhender les relations entre natures, sciences et sociétés, construisent et utilisent des cadres et des méthodes issus du dialogue entre différentes disciplines. Les chercheurs qui s'inscrivent dans ces communautés épistémiques conduisent des recherches qui mobilisent plusieurs disciplines, soit par des collaborations avec d'autres chercheurs soit en articulant eux-mêmes différents cadres de pensée et d'action. Un tel assemblage théorique et méthodologique réalisé par un chercheur ou un groupe de chercheurs est influencé par la communauté épistémique à laquelle se réfère sa recherche, mais il peut influencer tout autant l'identité de cette communauté que la manière dont s'y conçoit l'interdisciplinarité.

- Au pluralisme des sciences correspond une pluralité de constructions - théoriques et méthodologiques - qui permettent de valider un point de vue disciplinaire. S'il existe différents régimes de production de connaissances liés à la diversité des paradigmes mais aussi des objets et des méthodes, il est une nécessité commune aux sciences de proposer « leur vérité » au travers d'une construction qui ne fait sens qu'au sein de chacune d'elles. Ainsi, l'expérience interdisciplinaire apparaît bien comme une épreuve collective - de confrontation et de coopération entre différents régimes de production de connaissances - pour approcher la totalité du réel. Épreuve aujourd'hui réactivée par la montée en puissance des sciences dites «participatives » qui, audelà des savoirs scientifiques, interpellent d'autres formes de savoir (qu'ils soient issus de la pratique, de l'expérience, de l'action, etc.), non seulement pour les prendre en compte mais pour intégrer ceux qui les produisent dans un dispositif de recherche.

- Enfin, les besoins en formation sur les questions se situant à l'interface entre faits de nature et faits de société ont aussi poussé à la création de formations dans les universités et dans les grandes écoles dont le bilan et les perspectives méritent d'être soumis à l'examen, dix ans après la publication du numéro que NSS avait consacré à l'interdisciplinarité dans les formations ${ }^{6}$.

La Journée de l'association NSS-Dialogues du 8 janvier 2020 permettra de confronter les réflexions issues de ces quatre pistes de travail et de les mettre en débat avec tous les lecteurs de la revue qui souhaiteront nous rejoindre à cette occasion. Une journée qui veut s'inscrire dans l'esprit d'un commerce des idées.

Jean-Paul Billaud, Bernard Hubert, Franck-Dominique Vivien

\footnotetext{
${ }^{6}$ Jollivet M., Carlander M.-A. (Eds), 2007. Les formations interdisciplinaires: problèmes, expériences, perspectives, Natures Sciences Sociétés, 16, Supplément, https://doi.org/ 10.1051/nss:2008047.
} 